\title{
Serial position and the "labor-in-vain" effect
}

\author{
RICHARD KRINSKY \\ University of Southern Colorado, Pueblo, Colorado
}

\begin{abstract}
Serial input position functions were derived for means of the median self-paced study latencies and corresponding mean probabilities of recall memory for 27 consonant trigrams, whose overall means were previously reported by Nelson and Leonesio (1988, Experiment 1). Results of this analysis suggest that subjects "laboring" under accuracy instructions show a steeper linear rate of decrease in allocation of study times across serial input positions than do subjects laboring under speed instructions. Linear decreases in study times correspond to curvilinear (primacy and recency) changes in probability of recall memory. These data are consistent with previous research demonstrating the facilitation and attenuation of primacy and recency effects. These data are also consistent with notions of a memory system that allows for the monitoring of mnemonic success and allocation of effort on an item-per-item basis.
\end{abstract}

Nelson and Leonesio (1988) reported that when undergraduate subjects were asked to learn, either accurately or quickly, a list of items they had previously ranked with respect to ease of learning, the accuracy-emphasis group allocated more than twice as much self-paced study time (in learning a list of trigrams, Experiment 1) to more than seven times as much self-paced study time (in learning a list of general information questions, Experiment 3). However, this was a "labor in vain," because the two groups had little or no difference in recall (no more than $8 \%$ in any of the three experiments).

Lack of mastery during both self-paced and experimenterpaced study has previously been reported. For instance, in a free-recall experiment by Zimmerman (1975), the amount of self-paced study time was $13.6 \mathrm{sec}$ for two massed repetitions versus $8.9 \mathrm{sec}$ for a single repetition; the percentage of subsequent recall was $34 \%$ versus $32 \%$. Stoff and Eagle (1971) found that $1 \mathrm{sec}$ versus $3 \mathrm{sec}$ of experimenter-paced study time had no effect on subjects who reported using a rote-repetition strategy $(35 \%$ versus $36 \%$ recall).

One hypothesis for the lack of mastery by the accuracyemphasis group would be that they employed a displacedrehearsal mnemonic strategy during encoding (Hall, 1992; Rundus, 1971). Rundus (1971) demonstrated that subjects will often divert attention during study of to-be-recalled words from a current word to one that had been previously presented. The notion that subjects in the accuracyemphasis group were employing a displaced-rehearsal strategy gains further support because in Nelson and Leonesio's (1988) experiments, "subjects were instructed to rehearse each item silently while it was on the screen" (p. 678), thus allowing the subjects time to rehearse both current and previous trigrams.

This research was partially supported by a grant from the University of Southern Colorado. Correspondence should be addressed to Richard Krinsky, Psychology Department, University of Southern Colorado, Pueblo, CO 81001 .
Given that subjects employing rote memory during encoding were monitoring the success (and eventual futility) of that strategy, results of their recall efforts should be straightforward showing a primacy effect. Glanzer and Cunitz (1966) demonstrated that words presented quickly showed poorer primacy than words that were presented slowly. It would be reasonable to assume that since $\mathrm{Nel}-$ son and Leonesio (1988, Experiment 1) instructed their subjects to either study the items slowly (in the accuracy group) or quickly (in the speed group), there should be a corresponding primacy advantage for subjects acquiring items accurately.

Another line of more recent research also argues for a facilitation of primacy for subjects self-pacing their study under mastery-level instructions. Hall (1992) demonstrated that recall for words consecutively repeated (massed repetition) is equivalent to recall for words presented singly for an equivalent period of time. Previous research has demonstrated that subjects' attention lessens when words are presented as massed repetitions (Shaughnessy, Zimmerman, \& Underwood, 1972), allowing more time for rehearsal of earlier list items. Therefore, in Nelson and Leonesio's (1988) Experiment 1, subjects studying under accuracy instructions might have responded to prolonged self-paced presentations of items as though they were massed presentations.

Finally, it is also well known that items presented at the end of a list have a relatively high probability of being recalled (i.e., recency). Performing $30 \mathrm{sec}$ of arithmetic immediately following the study phase of a recall memory experiment attenuates recency (Glanzer \& Cunitz, 1966; Postman \& Phillips, 1965). Since Nelson and Leonesio (1988, Experiment 1) attempted to block recall from short-term memory by intrusion of arithmetic problems, it would be reasonable to assume that recency effects between speed and accuracy groups would be negligible.

Although predictions regarding serial position functions for the recall probabilities of speed and accuracy groups are straightforward, serial position functions for self-paced 
study latencies are not. It may be the case that subjects acquiring trigrams under speed instructions would show a more constant rate of study, allocating less time for rehearsal and memory monitoring, than subjects acquiring trigrams under accuracy instructions. For subjects given accuracy instructions, we might predict increased selfpaced study latencies across initial serial input positions as attempts to rehearse current and previous items continue. However, once the futility of that strategy is recognized (the subject realizes that he/she has already forgotten one or more of the items), then allocation of study time might reach asymptote and decline because of diminished attention for all remaining items. To validate our serial position hypotheses, data originally summarized by Nelson and Leonesio (1988, Experiment 1) was reexamined and subjected to a finer serial input position analysis. ${ }^{1}$

\section{METHOD}

Nelson and Leonesio (1988, Experiment 1) reported overall differences between the mean probability of recall and the mean of the medians for self-paced study latencies between speed and accuracy groups. Their data were, however, individually coded on an item-per-item basis, and we were, therefore, able to examine allocations of self-paced study times and corresponding probabilities of correct recall for each serial position. All serial position latency time measures and corresponding recall memory scores for 108 University of Washington undergraduates were input to a PRIME 9955 mainframe computer, and all subsequent data analyses were conducted via MINITAB.

\section{RESULTS AND DISCUSSION}

Averages of median self-paced study latencies and average probabilities of correct recall, collapsed across every three consecutive serial input positions for speed and accuracy groups, are displayed in Figure 1.

Visual inspection of these functions suggests that linear decreases in self-paced study times, across serial input positions, are curvilinearly related to probabilities of correct recall. As expected, subjects acquiring items under accuracy appear to allocate more time and show greater probability of recall for those items in the first versus the middle or last serial input positions. These apparent trends for mean study times and for mean probabilities of correct recall were validated by standard parametric statistical procedures.

Separate repeated measures, split plot analyses of variance, with the probability of a Type 1 error set at .05 , were conducted for differences between means of the median study times and mean probabilities of correct recall across the averaged nine serial input positions for speed and accuracy groups. As previously reported by Nelson and Leonesio (1988, Experiment 1), the overall means of the median self-paced study times were 1.9 and $5.4 \mathrm{sec}$ for the speed and accuracy groups, respectively.

The overall return in mean probability of correct recall for study-time investment was $49 \%$ for the accuracy group and $43 \%$ for the speed group. In other words, as described by Nelson and Leonesio (1988, Experiment 1),
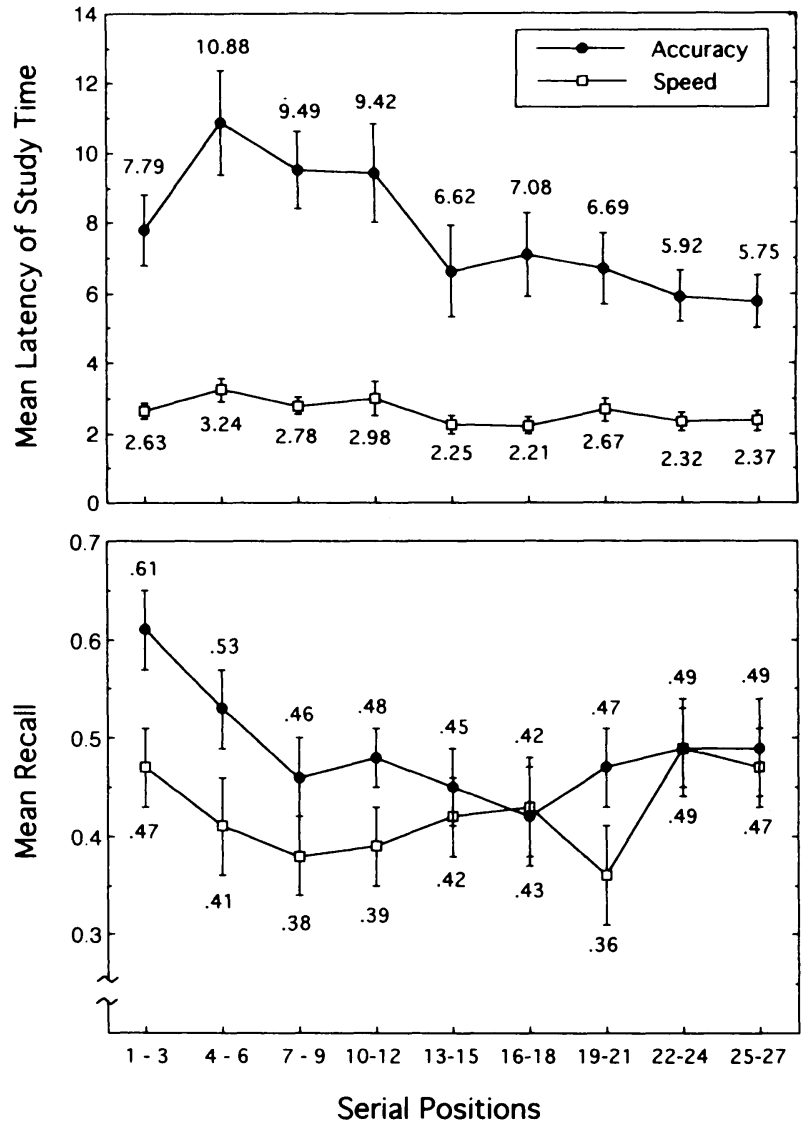

Figure 1. Averaged serial input position functions for means and standard errors of the median self-paced study latencies and mean probabilities of correct recall for subjects studying 27 trigrams under speed and accuracy instructions.

the accuracy group invested approximately $185 \%$ more study time for a $6 \%$ return in recall memory.

As predicted, from a displaced-rehearsal model, mean allocations of study time and mean probabilities for correct recall varied across serial input positions. Statistically reliable main effects for differences between serial input positions (averaged across experimental groups) did not occur for means of median study-time latencies $[F(8,848)$ $\left.=6.74, M S_{\mathrm{e}}=17.9\right]$ or for mean probabilities of correct recall $\left[F(8,848)=2.49, M S_{\mathrm{e}}=0.077\right]$. A finer trend analysis yielded a reliable linear trend for studytime means $\left[F(1,848)=28.00, M S_{\mathrm{e}}=17.9\right]$ and a reliable quadratic (primacy and recency) trend for mean probabilities of correct recall $\left[F(1,848)=15.96, M S_{\mathrm{e}}=\right.$ 0.076]. Also, the linear component of study-time means for speed versus accuracy groups interacted reliably across serial input positions $\left[F(8,848)=14.23, M S_{\mathrm{e}}=17.9\right]$. However, a reliable quadratic and serial input position interaction was not detected.

Reliable differences in allocation of study times between speed and accuracy groups occurred in conjunction with 
reliable differences in mean probabilities of correct recall across the first six list items (first and second averaged serial input positions in Figure 1). Accuracy subjects allocated an average of $7.79 \mathrm{sec}$ of study time to each of the first three list items, and speed subjects allocated an average of $2.63 \mathrm{sec}$. The 5.16-sec difference between those means was highly reliable $[t(106)=4.89, p<$ $.0001]$. The corresponding mean percentage of recall for the accuracy group was $61 \%$, compared with $47 \%$ for the speed group. The $14 \%$ difference between those means was also statistically reliable $[t(106)=2.30, p<.05]$. Therefore, subjects "laboring" under accuracy instructions allocated approximately $195 \%$ more study time than did subjects laboring under speed instructions, and this extra output of effort yielded a $30 \%$ proportional return in recall memory.

The same pattern of results occurred for the second averaged serial input position. Accuracy subjects allocated an average of $10.88 \mathrm{sec}$ of study time across the fourth, fifth, and sixth list items. Speed subjects, however, only allocated $3.24 \mathrm{sec}$. The difference between those means was statistically reliable $(t=4.98, p<.0001)$. Accuracy subjects recalled $53 \%$ of those list items correctly, compared with $41 \%$ for speed subjects. The $12 \%$ difference between those mean percentages was also statistically reliable $[t(106)=1.96, p<.05]$. Therefore, at the second averaged serial input position, a $235 \%$ increase in study time for accuracy-instructed subjects relative to speed-instructed subjects yielded a $30 \%$ proportional return in recall.

Although accuracy-instructed subjects allocated reliably more average study time than did speed-instructed subjects at all remaining serial input positions, reliable differences between mean probabilities for recall at all subsequent serial positions were not detected. After approximately the 3rd average serial input position (9th list item), large proportional between-group allocations of study time were accompanied by very small between-group differences in recall memory, thereby producing what Nelson and Leonesio (1988) referred to as a "labor-in-vain" effect. For example, across the 16th, 17th, and 18th list items (6th average serial input position), subjects laboring under accuracy instructions allocated approximately $220 \%$ more study time than did subjects laboring under speed instructions, and they recalled $2 \%$ fewer items. Across the 22nd, 23rd, and 24th list items (8th average serial input posi- tion), a $155 \%$ proportional increase in study time was associated with a $0 \%$ increase in recall memory.

\section{GENERAL DISCUSSION}

In summary, our serial input position analysis confirmed the following hypotheses: (1) allocation of study time is greater for those items that occur early in the list compared with those that occur later in the list, and (2) increases in study time are associated with increases in the probability of recall for those items that also occur early.

These results are straightforward from a displaced-rehearsal (rote memory) model of study and review. This model would predict that subjects "laboring" for accuracy would divert their attention from prolonged presentations of current trigrams in order to gain a rehearsal advantage for previous trigrams. The subjects may have monitored the futility of their efforts (durability of recall for previous items, but forgetting of current items) after approximately the ninti list item, and then hurried through the remaining items as a result of diminished attention to the task at hand. In other words, subjects studying under accuracy instructions, in Nelson and Leonesio's (1988) Experiment 1, may have been able to monitor their "labor in vain" and modify their subsequent effort accordingly.

\section{REFERENCES}

Glanzer, M., \& Cunitz, A. R. (1966). Two-storage mechanisms in free-recall. Journal of Verbal Learning \& Verbal Behavior, 5, 351-360.

HaLL, J. W. (1992). Unmixing effects of spacing on free recall. Journal of Experimental Psychology: Learning, Memory, \& Cognition, 18, 608-614.

Nelson, T. O., \& LEONESIO, R. J. (1988). Allocation of self-paced study time and the "labor-in-vain effect." Journal of Experimental Psychology: Learning, Memory, \& Cognition, 14, 676-686.

Postman, L., \& Phillips, L. W. (1965). Short-term temporal changes in free-recall. Quarterly Journal of Experimental Psychology, 17, 132-138.

RUNDUS, D. (1971). Analysis of rehearsal processes in free recall. Journal of Experimental Psychology, 89, 63-77.

Shaughnessy, J. J., Zimmerman, J., \& UNDERwoOd, B. J. (1972). Further evidence on the MD-DP effect in free recall learning. Journal of Verbal Learning \& Verbal Behavior, 11, 1-12.

STOFF, D. M., \& EAGLE, M. N. (1971). The relationship among reported strategies, presentation rate, and verbal ability and their effects on free recall learning. Journal of Experimental Psychology, 87, 423-428.

Zimmerman, J. (1975) Free recall after self-paced study: A test of the attention explanation of the spacing effect. American Journal of Psychology, 88, 227-291.

\section{NOTE}

1. Data for reanalysis were derived from protocols provided by Thomas O. Nelson.

(Manuscript received April 2, 1993.) 